\title{
CALCULATIONS OF ELECTRON ACCUMULATION IN THE SNS STORAGE RING
}

\author{
V.Danilov, A.Aleksandrov, ORNL SNS Project, Oak Ridge, TN \\ M. Blaskiewicz, BNL, Brookhaven, NY, J. Wei, BNL/ORNL, Brookhaven, NY
}

\begin{abstract}
This is a study to determine what kind of materials could be used in the Spallation Neutron Source ring to prevent electron accumulation due to the secondary emission. The calculations presented here are simple but based on experimental facts. Practical recommendations for the accumulator ring are considered.
\end{abstract}

\section{INTRODUCTION}

For high intensity proton machines, electron cloud formation may lead to instabilities. Proton beam instabilities have been observed at LANL PSR [1], AGS Booster in BNL [2], KEK Booster [3], and CERN PS [4]. A probable explanation of these instabilities is that there exists a large electron density in the vacuum chamber that interacts with the proton beam. This leads to a transverse mode coupling instability between the circulating protons and oscillating electrons trapped in the proton potential well. Multipacting drastically increases the electron density, which, in turn, leads to the instability. The purpose of this paper is to ascertain the risk of electron accumulation in the SNS ring.

The phenomenon of electron accumulation is described in [5]. We are interested here in electron accumulation for the bunched beam mode. For bunched beams there are two scenarios, single pass and multi-pass, for electron accumulation. These bunched beam scenarios are presented in the following sections.

\section{DETERMINATION OF THE THRESHOLD SEM COEFFICIENT}

\subsection{Single Pass Electron Accumulation}

The first mechanism of electron accumulation is related to multipacting at the trailing edge of the proton beam. On the trailing edge of the proton bunch, the longitudinal bunch density is decreasing causing the electrons to gain energy. If there are many electrons from beam losses at the longitudinal center of the bunch, or if the secondary emission coefficient is large, the electron cloud density could reach the density of the proton beam. Almost all electrons accumulated in a single pass disappear in the beam gap due to their own space charge. These effects are estimated in this section.

To model this scenario, a computer code was created that calculates 1D electron trajectories, starting from the vacuum chamber wall. After an electron strikes the wall, secondary electrons are emitted depending on the initial energy of the primary electron. The secondary electrons oscillate in the proton potential, gaining energy at the rate of about $100 \mathrm{eV}$ per traversal of the vacuum chamber.

Figure 1 shows an example of electron motion (solid line) with respect to the proton bunch distribution (dashed line). The zero longitudinal coordinate corresponds to the center of the beam gap, the initial electron vertical coordinate is close to the vacuum chamber radius of $5 \mathrm{~cm}$, and the initial distance between the gap and the electron is 38 meters. Initially the electron oscillation amplitude decreases due to the increasing proton beam density. Once the proton beam center has passed the electron, the transverse amplitude increases and the electron finally hits the wall several times, losing all its energy with each impact. In the model, the total number of secondary emission electrons is summed over all collisions with the vacuum chamber using the formula for secondary emission yield $y(E)$ from Reference [6] and assuming the primary electrons are normal to the surface. That is:

$$
y(E)=y_{\max } 1.11\left(E / E_{m}\right)^{-.35}\left(1-e^{-2.3\left(E / E_{m}\right)^{1.35}}\right)
$$

where $E_{m}=400 \mathrm{eV}$ and $y_{\max }$ depends on the vacuum chamber material. The proton beam transverse distribution is taken to be constant within the beam radius, and equal to zero otherwise.

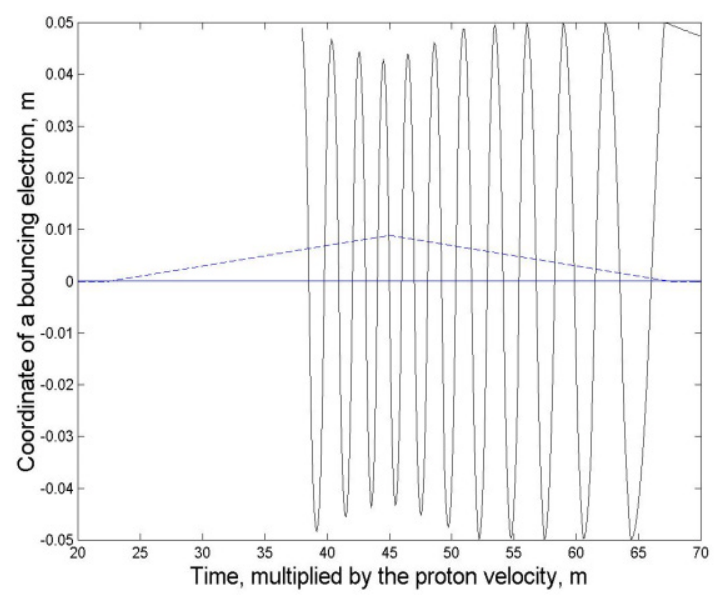

Figure 1 Coordinate of an electron oscillation in the electric field of proton beam.

Figure 2 shows the PSR and the SNS Trailing Edge Multiplication Parameters (TEMP), defined as the logarithm of number of electrons at the trailing edge produced by one electron starting from the vacuum chamber at the center of the proton bunch. The plot shows 
the parameters for various shapes of the SNS beam (the parameters are shown in Table 1). The dotted blue line and the dashed red line show TEMP for PSR for SNS, respectively, with both calculated for triangular distributions of the proton beam. The three solid lines correspond to various trail distribution functions, in the form of $C \cdot\left(1-\frac{s^{2}}{L_{b}{ }^{2}}\right)^{\mu}$, where the constant parameter

Table 1: SNS and PSR parameters for simulations

\begin{tabular}{|l|c|c|}
\hline Parameters & SNS & PSR \\
\hline $\begin{array}{l}\text { Proton kinetic } \\
\text { energy, GeV }\end{array}$ & 1 & 0.8 \\
\hline Perimeter, $\mathrm{m}$ & 248 & 90 \\
\hline $\begin{array}{l}\text { Beam length, } \\
\mathrm{m}\end{array}$ & 160 & 60 \\
\hline $\begin{array}{l}\text { Number of } \\
\text { protons }\end{array}$ & $2 * 10^{14}$ & $3 * 10^{13}$ \\
\hline $\begin{array}{l}\text { Chamber } \\
\text { radius, cm }\end{array}$ & 10 & 5 \\
\hline $\begin{array}{l}\text { Beam rms } \\
\text { radius, cm }\end{array}$ & 2 & 2 \\
\hline
\end{tabular}

$\mathrm{C}$ is adjusted to keep the number of particles constant, independent of $\mu$. The distribution function becomes smoother with increasing $\mu$. The lower solid line shows TEMP for $\mu=1$, which corresponds to a parabolic distribution. The middle and upper curves correspond to $\mu=2$ and $\mu=5$, respectively. The TEMP Parameter increases with increasing smoothness, even exceeding one for the triangular distribution (shown as the red dashed line).

Figure 2 shows that TEMP is much larger for SNS than for PSR. This is related to the fact that the SNS ring has a larger pipe aperture (10 cm against $5 \mathrm{~cm}$ for PSR) and more intense longer pulse than PSR. The amplification of the electron density occurs for the triangular distribution when the maximum SEM coefficient exceeds 1.8. For the coefficient of 2, the multiplication factor is about $\mathrm{e}^{10}$. This could lead to $100 \%$ compensation of the proton density by the electrons, and can be regarded as a threshold value for the appearance of large electron density (see, e.g.[7]) for the triangular longitudinal distribution of the proton bunch. Other longitudinal distributions have different values for the threshold TEMP. Thus, TEMP has a strong dependence on the proton bunch distribution, which is a typical feature of the e-p instability. For large maximum SEM coefficients ( $y_{\max }$ in (1)) the TEMP is roughly proportional to $\left(\mathrm{y}_{\max }\right)^{1 / 3}$.

\subsection{Multi-Turn Electron Accumulation}

Multi-turn electron accumulation is more complicated than single pass accumulation. It significantly depends not only on the SEM coefficient but also on the energy and angle distribution of the secondary electrons, the proton beam gap length, the amount of beam in the gap, etc. A simple model can be used to explain the main idea. Assume that the proton beam has a rectangular longitudinal distribution and a uniform transverse distribution (see Figure 3).

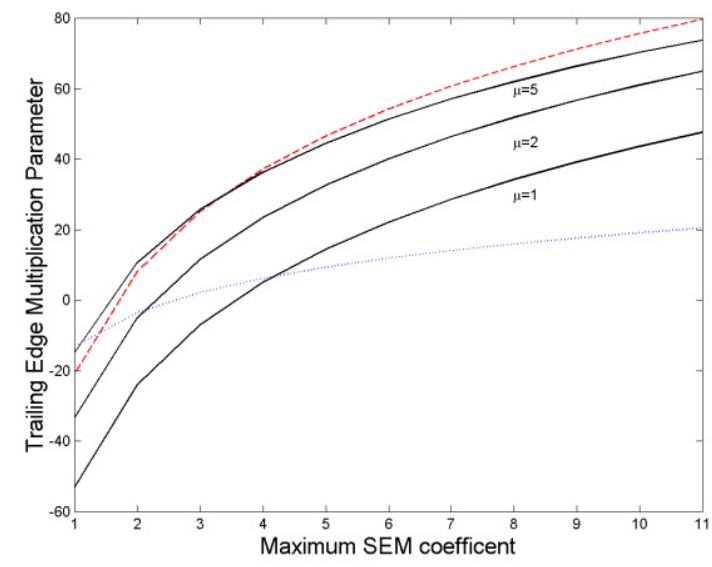

Figure 2 Trailing Edge Multiplication Parameter versus the maximum SEM coefficient of various materials. Solid line is for the SNS ring, dashed blue line - for PSR.

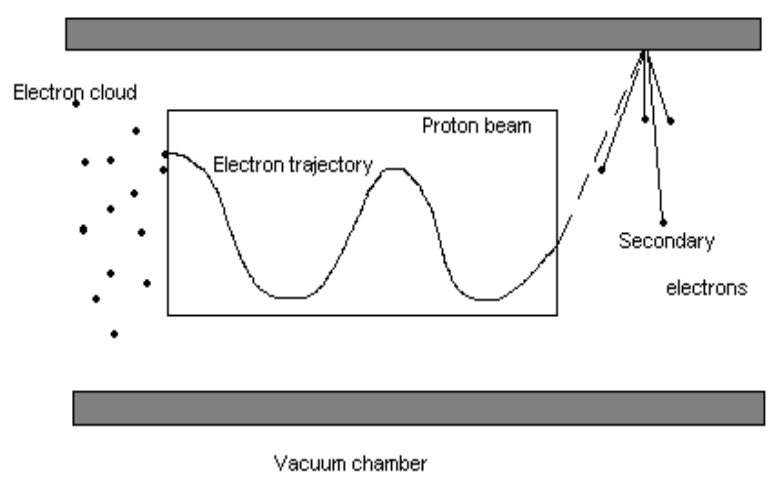

Figure 3 Schematic of electron motion in the field of the proton bunch.

The electron energies outside the proton beam are determined by the energy distribution of the secondary emission electrons. Since these energies are small compared to the electric potential of the proton beam, inside the proton beam the electrons oscillate according to the cosine law and have the maximum kinetic energies up to several $\mathrm{keV}$, depending on the beam intensity. For example, the electric potential at the center of the PSR proton beam is about $5 \mathrm{kV}$. At the end of the proton beam the frequency and the bunch length determine the electron transverse oscillation phase. For a 90-degree electron phase advance, the electron energy at the end would be equal to the electric potential, more than enough to produce the secondary electrons. Some of these particles survive the beam gap and repeat the described process again. When the electron cloud density is big enough to repel the secondary particles back into the wall, the accumulation saturates. 
A computer code was written to study both the effects of gap survival and the electric field generated by the electrons. The code assumes that the proton beam and electron cloud are both cylindrically symmetric within a round, straight section, vacuum chamber. Longitudinal electric fields are ignored, since they produce velocities small compared to the beam velocity. The electron phase space distribution is modeled as a sum of cylindrical shells. The acceleration of shell $j$ due to shell $k$ is taken to be nonzero only if $r_{k}<r_{j}$. In this case the acceleration is $\left(\mathrm{q}_{\mathrm{e}} / 2 \pi \varepsilon_{0} \mathrm{~m}_{\mathrm{e}}\right) \lambda_{\mathrm{k}} \mathrm{r}_{\mathrm{j}} /\left(\mathrm{d}^{2}+\mathrm{r}_{\mathrm{j}}^{2}\right)$, where $\lambda_{\mathrm{k}}$ is the charge per unit length on shell $k$. The smoothing length $d$ is of order 1 millimeter, which is small compared to the $\sigma=8$ millimeter rms beam size. The electric field due to the beam has the same form as that for the electrons with the beam at $r=0, \lambda_{\mathrm{e}}$ replaced by the instantaneous proton line density $\lambda_{\mathrm{p}}$, and $\mathrm{d}^{2} \rightarrow 2 \sigma^{2}$. We assume $\mathrm{E}_{\max }=300 \mathrm{eV}$ and also allow for electron reflections. The reflection coefficient versus impact energy is given by $R(E)=R_{0}$ $\exp (-\mathrm{E} / 50 \mathrm{eV})$. Electrons are created at the wall with a generation rate proportional to the instantaneous proton line density. The time average generation rate is $2 * 10^{7} \sim$ electrons/meter/turn and the simulation results saturate for larger production rates.

Tables 2 and 3 show the results for PSR and SNS, respectively. The minimum value of the electron line density, $\min \left(\lambda_{\mathrm{e}}\right)$, is that which survives the gap, and is an appropriate input parameter for a wakefield description of the e-p instability. Notice that $\min \left(\lambda_{\mathrm{e}}\right)$ depends strongly on $\mathrm{R}_{0}$, but weakly on both the bunch shape and the secondary emission yield. It also depends on gap length. $\mathrm{Q}_{\text {wall }}$ stands for the integrated charge that strikes the vacuum chamber per turn per $\mathrm{cm}^{2}$. The variation of other parameters is similar to that in the simple model described earlier.

Table 2 Steady state conditions for PSR with $7 \mu \mathrm{C}$. $\max \left(\lambda_{\mathrm{p}}\right)=208 \mathrm{nC} / \mathrm{m}$ for $\mu=1$ and $260 \mathrm{nC} / \mathrm{m}$ for $\mu=2$.

\begin{tabular}{|l|l|l|l|l|}
\hline$\mu$ & $\mathrm{R}_{0}$ & $\mathrm{Y}_{\max }$ & $\begin{array}{l}\mathrm{Q}_{\text {wall }} \\
\mathrm{PC} / \mathrm{cm}^{2}\end{array}$ & $\begin{array}{l}\min \left(\lambda_{\mathrm{e}}\right) \\
\mathrm{nC} / \mathrm{m}\end{array}$ \\
\hline 1 & 0 & 2.5 & 0.24 & 0.05 \\
\hline 2 & 0 & 2.5 & 2.4 & 0.07 \\
\hline 1 & 0 & 3.0 & 1.6 & 0.09 \\
\hline 2 & 0 & 3.0 & 11.5 & 0.08 \\
\hline 1 & 0.5 & 2.5 & 0.42 & 0.25 \\
\hline 2 & 0.5 & 2.5 & 2.4 & 0.52 \\
\hline 1 & 0.5 & 3.0 & 1.6 & 0.52 \\
\hline 2 & 0.5 & 3.0 & 11.7 & 0.56 \\
\hline
\end{tabular}

\section{PRACTICAL CONSEQUENCES}

The calculated threshold SEM coefficient was estimated to be in the range 1.8-2. Consequently, every piece of the vacuum chamber should be coated with TiN, which has a maximum SEM of about 1.5. Because of convolutions (see e.g. [8]), the unshielded bellow coefficient could be about $20 \%$ higher than that number. If the bellow surface is coated with TiN and is well conditioned (the maximum
SEM is about 1.5), one can expect the resulting coefficient not to exceed 1.8. Thus, it is likely that the SNS ring will not undergo electron cloud build-up in the bellows. However, since 1.8 is a marginal value, it is worthwhile to install electron detectors to check this conclusion. In addition, the ring will have a relatively high vacuum $\left(5 \times 10^{-9}\right.$ Torr $)$ to reduce the initial electron density, a beam-in-gap kicker to reduce number of electrons surviving the gap, and an electron collector near the stripping foil.

Table 3 Steady state conditions for SNS with $32 \mu \mathrm{C}$. $\max \left(\lambda_{\mathrm{p}}\right)=300 \mathrm{nC} / \mathrm{m}$ for $\mu=1$ and $375 \mathrm{nC} / \mathrm{m}$ for $\mu=2$.

\begin{tabular}{|l|l|l|l|l|}
\hline$\mu$ & $\mathrm{R}_{0}$ & $\mathrm{Y}_{\max }$ & $\begin{array}{l}\mathrm{Q}_{\text {wall }} \\
\mathrm{PC} / \mathrm{cm}^{2}\end{array}$ & $\begin{array}{l}\mathrm{min}\left(\lambda_{\mathrm{e}}\right) \\
\mathrm{nC} / \mathrm{m}\end{array}$ \\
\hline 2 & 0 & 2.0 & 6.6 & 0.05 \\
\hline 1 & 0 & 2.0 & 0.32 & 0.05 \\
\hline 2 & 0 & 2.5 & 67.2 & 0.054 \\
\hline 1 & 0 & 2.5 & 6.0 & 0.07 \\
\hline 2 & 0.5 & 2.0 & 5.9 & 0.5 \\
\hline 1 & 0.5 & 2.0 & 0.35 & 0.2 \\
\hline 2 & 0.5 & 2.5 & 75.0 & 0.50 \\
\hline 1 & 0.5 & 2.5 & 5.2 & 0.56 \\
\hline
\end{tabular}

\section{ACKNOWLEGMENTS}

The authors acknowledge support of the DOE, under contract no. DE-AC05-00OR22725 with UT-Batelle, LLC for ORNL, and are grateful to Robert Macek for providing information on the PSR instability and electron accumulation. We are grateful to Robert Kustom and David Olsen for their help and the support of all these activities and to Mike Plum, Tai-Sen Wang, and Paul Channell for valuable discussions and sharing their ideas on the e-p instability.

\section{REFERENCES}

[1] R. Macek,"Overview of the Program Plan and the Experimental Results from PSR", LBLN Workshop on the e-p instability, November, 20-21, 2000.

[2] M. Blaskiewicz, ibid.

[3] Y. Irie, et al, KEK Proc. 97-17, (1997) pp 247-255

[4] R. Cappi, G. Metral, could be found at http://www.cern.ch/PSdoc/ppc/md/md980716/epinstab.ht $\mathrm{ml}$

[5]A. Aleksandov, V. Danilov, M. Blaskiewicz. "Electron Accumulation in the PSR: An Attempt to Understand the Basic Facts", private communication.

[6] F. Zimmerman, "Electron-Cloud Instability and beam Induced Multipacting in the LHC and in theVLHC", SLAC-PUB-7664, October 1997.

[7] V. Danilov, et.al. , "Multipacting on the trailing edge of proton beam bunches in the PSR and SNS", Workshop on Instabilities of High Intensity Hadron Beams in Rings, Upton, New York, 1999

[8] R. Kirby, "Secondary Electron Emission From Accelerator Materials", presented at ICFA Workshop on Two-Stream Instabilities, Santa Fe, February 16-18, 2001. 